\title{
BIOCONTROL DEL MOHO FOLIAR DEL TOMATE CLADOSPORIUM FULVUM EMPLEANDO CUATRO HONGOS ANTAGONISTAS $\left({ }^{\mathbf{1}}\right)$
}

\author{
ENRIQUE TORRES $(2,4)$; JOSÉ IANNACONE $\left(2,3^{*}\right)$; HILDA GOMEZ $\left({ }^{4}\right)$
}

\begin{abstract}
RESUMEN
El moho foliar Cladosporium fulvum (Cooke 1883) produce una enfermedad que ataca el cultivo de tomate en invernadero en el Perú. Como alternativa al control de este patógeno se probó la eficiencia bajo condiciones in vitro e invernadero de cuatro hongos antagonistas: Hansfordia pulvinata (Berk y Curt 1958), Trichoderma harzianum (Rifai 1969), T. viride (Persoon 1821) y T. virens (Miller, Giddens y Foster 1963). El cultivo y crecimiento de los hongos se realizó en Agar Sabouraud para Trichoderma spp. y en Agar Jugo $\mathrm{V}_{8}$ para $H$. pulvinata. El bioensayo empleado fue el de placas precolonizadas propuesto por Krauss. En condiciones in vitro se observó que a $24^{\circ} \mathrm{C}$ y $72 \mathrm{~h}$, el crecimiento de T. harzianum fue estadísticamente diferente de las otras tres especies ensayadas, y a $28{ }^{\circ} \mathrm{C}$ fue similar a T. viride. A $24{ }^{\circ} \mathrm{C}$ y a las $120 \mathrm{~h}, \mathrm{~T}$. virens fue diferente de todas las especies evaluadas. A las $168 \mathrm{~h}$ el crecimiento de las tres especies de Trichoderma fue estadísticamente similar, diferenciándose de H. pulvinata. A $28{ }^{\circ} \mathrm{C}$ a las $120 \mathrm{~h}$ y $168 \mathrm{~h}$ los resultados fueron estadísticamente similares para las tres especies de Trichoderma, pero diferentes de H. pulvinata. El mecanismo de acción más común para los hongos antagonistas fue el micoparasitismo. Finalmente, $T$. harzianum fue más eficiente debido a que redujo la severidad de la enfermedad en un $19,35 \%$ bajo condiciones de invernadero.
\end{abstract}

Palabras clave: Cladosporium fulvum, control biológico, Hansfordia pulvinata, tomate, Trichoderma harzianum, Trichoderma viride, Trichoderma virens.

\section{ABSTRACT \\ BIOCONTROL OF LEAF MOLD OF TOMATO CLADOSPORIUM FULVUM (COOKE 1883) EMPLOYING FOUR ANTAGONISTIC FUNGI}

Leaf mold Cladosporium fulvum (Cooke 1883), produce a tomato crop disease under greenhouse in Peru. As an alternative to control this pathogen the efficiency under in vitro conditions and greenhouse conditions of four antagonistic fungi was probed: Hansfordia pulvinata Berk y Curt 1958, Trichoderma harzianum (Rifai 1969), T. viride (Persoon 1821) and T. virens (Miller, Giddens y Foster 1963). Fungal culture and growth was performed in Agar Sabouraud for Trichoderma spp. and in Agar Juice $\mathrm{V}_{8}$ for H. pulvinata. The bioassay employed was precolonized plates proposed by Krauss. In in vitro conditions was observed that at $24^{\circ} \mathrm{C}$ and $72 \mathrm{~h}$ growing of $T$. harzianum was statistically different than the other three species tested, and at $28^{\circ} \mathrm{C}$ was similar to T. viride. At $120 \mathrm{~h}$ and $24^{\circ} \mathrm{C}$ growing of T. virens was different from all species evaluated. At $168 \mathrm{~h}$ growing of the three species of Trichoderma, were statistically similar, varying from $H$. pulvinata. At $28^{\circ} \mathrm{C}$ and at $120 \mathrm{~h}$ and $168 \mathrm{~h}$ the results were statistically similar to the three species of Trichoderma, but different to H. pulvinata. Micoparasitism is the most common-action mechanism. Finally, T. harzianum was the most efficient antagonistic fungus because reduced disease in $19.35 \%$ under greenhouse conditions.

Key words: biological control, Cladosporium fulvum, Hansfordia pulvinata, tomatoes, Trichoderma harzianum, Trichoderma viride, Trichoderma virens.

( $\left.{ }^{1}\right)$ Recibido para publicación el 5 de mayo de 2004 y aceptado el 5 de julio de 2007.

( ${ }^{2}$ ) Universidad Nacional Federico Villarreal. Facultad de Ciencias Naturales y Matemáticas. Laboratorio de Ecofisiología Animal. Calle San Marcos 383, Pueblo Libre. Lima, Perú.

$\left({ }^{3}\right)$ Universidad Ricardo Palma. Facultad de Ciencias Biológicas. Av. Benavides s/n, Santiago de Surco. Lima, Perú. E-mail: joseiannacone@yahoo.es (*) Autor responsable.

(4) Laboratorio de Hongos Antagonistas. Programa Nacional de Control Biológico, Servicio Nacional de Sanidad Agraria (PNCB-SENASA). 


\title{
BIOCONTROLE DO MOFO FOLIAR EM TOMATEIRO EMPREGANDO QUATRO FUNGOS ANTAGONISTAS
}

\begin{abstract}
RESUMO
O mofo foliar Cladosporium fulvum (Cooke, 1883) produz uma enfermidade que ataca o cultivo do tomateiro em casa de vegetação no Peru. Como alternativa ao controle desse patógeno, testou-se a eficiência, sob condições in vitro e casa de vegetatção, de quatro fungos antagonistas: Hansfordia pulvinata (Berk y Curt 1958), Trichoderma harzianum (Rifai 1969), T. viride (Persoon 1821) y T. virens (Miller, Giddens y Foster 1963). O cultivo e crescimento dos fungos foi realizado em Agar Sabouraud para Trichoderma spp. e em Agar Suco V8 para H. pulvinata. O bioensaio empregado foi o de placas pré-colonizadas proposto por Krauss. Em condições in vitro, observou-se que a $24{ }^{\circ} \mathrm{C}$ e 72 horas, o crescimento de T. Harzinum foi estatisticamente diferente das outras três espécies estudadas, e a $28{ }^{\circ} \mathrm{C}$ foi semelhante a T. viride. A $24{ }^{\circ} \mathrm{C}$ e 120 horas, T. virens foi diferente de todas as espécies avaliadas. A 168 horas, o crescimento das três espécies de Trichoderma foi estatisticamente semelhante, diferenciando-se de H. pulvinata. A $28^{\circ} \mathrm{C}$ e 120 e 168 horas, os resultados foram estatisticamente semelhantes para as três espécies de Trichoderma, mas diferentes de H. pulvinata. O mecanismo de ação mais comum para os fungos antagonistas foi o micoparasitismo. Finalmente, T. harzianum foi mais eficiente devido à redução da severidade da enfermidade em $19,35 \%$ sob condições de casa de vegetação.
\end{abstract}

Palavras-chave: Cadosporium fulvum, controle biológico, Hansfordia pulvinata, tomate, Trichoderma harzianum, Trichoderma viride, Trichoderma virens.

\section{INTRODUCCIÓN}

El tomate (Lycopersicum esculentum Mill) es una de las especies agrícolas más conocidas y exportadas comercialmente a nivel mundial. Esta especie es una de las hortalizas de mayor importancia para el consumo humano directo (IANNACONe y ReYes, 2001; De Wit et al., 2002; IAnNacone y Murrugarra, 2002).

Dentro del aspecto fitosanitario, el cultivo del tomate es afectado por diversas plagas y enfermedades. Los patógenos que afectan la parte aérea del tomate son muy diversos y variados, muchos de ellos pueden ocasionar pérdidas muy severas si no se toman las medidas de control adecuadas (APAZA, 1999; REY et al., 2000). Una de estas enfermedades es la cladosporiosis, conocida como Moho foliar del tomate, cuyo agente etiológico es Cladosporium fulvum (Cooke, 1883; sin. Fulvia fulva), (GABOR y Wiebe, 1997; JoOsten y De Wit, 1999). El moho foliar es una enfermedad que normalmente se encuentra atacando este cultivo bajo condiciones de invernadero (ELAD, 2003). En ataques graves gran parte de la superficie foliar queda inutilizada para realizar la fotosíntesis, lo que se traduce en un descenso en el rendimiento y en la calidad del fruto (De W IT et al., 2002).

Una alternativa dentro del control biológico es el empleo de hongos antagonistas, cuyo conocimiento de su efectividad y de su mecanismo de acción es esencial para el desarrollo de una estrategia en la implantación de una agricultura sostenible (Perese Y Le Picard, 1980; Honee et al., 1994; Martinez et al., 1994; Guarro et al., 1999; LieCKFELdT et al., 1999; SelitrenNiKOFF, 2001; Williams et al., 2003). El control biológico utilizando micoparásitos, así como cepas fúngicas del genero Trichoderma para el control de enfermedades de plantas producidas por hongos, constituye hoy en día una alternativa que podría sustituir el control químico sintético, que además de su elevado costo, trae como consecuencia el desarrollo de resistencia por parte del fitopatógeno, y problemas de contaminación y toxicidad (Grondona et al., 1997; CAstle et al., 1998; Rey et al., 2000; RodríGuez, 2002). Además es una estrategia que está dando buen resultado contra los agentes infecciosos al desplazar a éstos de manera natural (PAPAvizas, 1985; Hermosa et al., 2000; Rey et al., 2000; SANTAMARINA et al., 2002).

Diferentes especies del género Trichoderma han sido usadas como agentes de control biológico de enfermedades en la agricultura (Lo et al., 1997; KLEIN y Eveleigh, 1998; Hermosa et al., 2000; Sariah et al., 2005). Los más utilizados son Trichoderma harzianum (Rifai 1969), T. viride (Persoon 1829) y T. virens (J. Miller, Giddens \& Foster 1963) (SAmuELs, 2004). Se ha demostrado que son capaces de controlar significativamente bajo condiciones in vitro a hongos de diversos géneros, como Armillaria (Vahl), Botrytis (Persoon 1801), Chondrostereum (Persoon 1801), Colletotrichum (Sacc 1901), Diaporthe (Wolf), Endothia (Murris), Fulvia (Ciferreri), Fusarium (Link ex Gray 1821), Fusicladium (Sacc 1901), Helminthosporium (Link 1821), Pseudoperonospora (Berk \& Curt), Pythium (Pringsh), Rhizoctonia (Kühn), Rhizopus (Corda 1838), Sclerotinia (Sacc 1901), Sclerotium (Sacc 1901), Venturia (Sacc 1901) y Verticillium (Nees 1817) (GuARro et al., 
1999; Hermosa et al., 2000; Santamarina et al., 2002; Gielen et al., 2004; SAMuels, 2004). Además, Hansfordia pulvinata Berk y Curt 1958, se le ha encontrado parasitando al hongo C. fulvum en cultivo de tomate (Peresse y Le Picard, 1980).

Los objetivos específicos de la presente investigación fueron: 1) evaluar la efectividad y el tipo de acción de cuatro especies de hongos antagonistas: Hansfordia pulvinata, Trichoderma harzianum, T. viride y $T$. virens sobre $C$. fulvum en condiciones in vitro, 2) determinar la temperatura óptima a la cual se observa el mejor efecto de los cuatro hongos antagonistas frente al patógeno C. fulvum bajo condiciones in vitro, y 3) identificar el efecto de cuatro hongos antagonistas sobre la severidad de la enfermedad cladosporiosis, bajo condiciones de invernadero.

\section{MATERIAL Y MÉTODOS}

El presente trabajo de investigación se realizó durante los meses de agosto del 2003 a febrero del 2004 en el Laboratorio de Hongos Antagonistas y en el invernadero del Programa Nacional de Control Biológico- Servicio Nacional de Sanidad Agraria (PNCB- SENASA), con sede en Vitarte, Lima, Perú.

\section{Material biológico}

Se utilizaron cuatro cepas de hongos antagonistas, proporcionadas por Laboratorio de Hongos antagonistas del PNCB-SENASA y una cepa patogénica $C$. fulvum, que se aisló a partir de hojas con signos de la enfermedad (Tabla 1).

\section{Cladosporium fulvum}

Prueba de patogenicidad: Con la finalidad de reactivar y aislar el patógeno se procedió a realizar una prueba de patogenicidad. Se asperjó $50 \mathrm{~mL}$ de una solución madre de $10^{8}$ esporas $\mathrm{mL}^{-1}$ del cultivo de C. fulvum procedente de Ica sobre folíolos de plantas sanas de tomate, las cuales fueron incubadas a temperatura ambiente (24 ${ }^{\circ} \mathrm{C}$ a $28^{\circ} \mathrm{C}$ ) y $70 \%$ a $85 \%$ de humedad relativa (HR) hasta la aparición de los primeros síntomas ocasionados por el patógeno (a los 14 a 16 días). Seguidamente se recolectaron las hojas que presentaron el daño, y se transportaron al laboratorio para identificar el patógeno. La identificación de C. fulvum se basó en la morfología en cultivo, en las características de las conidias y del micelio, y en la sintomatología del patógeno en el cultivo de tomate (THomma et al., 2005). Se procedió a su reaislamiento por una puntura a un medio de cultivo a base de Agar de Sabouraud (MerCK, 2000). Se incubaron a $24^{\circ} \mathrm{C}$ y $60 \%$ de HR durante 15 días hasta que la superficie del medio fue colonizada por el patógeno. No se realizó ninguna determinación de razas fisiológicas de C. fulvum empleando técnicas moleculares (THOMmA et al., 2005).

Multiplicación: El hongo C. fulvum fue sembrado y multiplicado a partir de $10 \mathrm{~mL}$ de una solución madre. De esta dilución, se tomaron 7 gotas, las cuales se añadieron a placas de Petri, con el fin de extender el inóculo por toda el área de la placa utilizando el asa de Drigalski. Las placas contenían un medio de Agar de Sabouraud Dextrosa + Glucosa (MERCK, 2000); seguidamente fueron llevados a la incubadora a $24^{\circ} \mathrm{C}$ y $65 \%$ de HR por un periodo de 15 días hasta que la superficie del medio fue cubierta totalmente por el hongo patógeno. Al tener las placas totalmente colonizadas, éstas se utilizaron para la confrontación con los hongos antagonistas.

\section{Hongos antagonistas}

Multiplicación: Para multiplicar los hongos antagonistas $T$. harzianum, $T$. viride y $T$. virens, se cultivaron por puntura en placas Petri con Agar Sabouraud Dextrosa. El hongo H. pulvinata por sus requerimientos fue sembrado por el método de agotamiento y en un medio $\mathrm{V}_{8}$. Se incubaron por 10 días a $24^{\circ} \mathrm{C}$ y $28^{\circ} \mathrm{C}$ con $65 \%$ de HR. A partir de estas placas se tomaron tiras de cultivo de $5 \times 25 \mathrm{~mm}$ que fueron colocadas en el interior de las placas precolonizadas con C. fulvum y se incubaron durante 7 días, donde se evaluó la interacción antagonistapatógeno. Las tres especies de hongos antagonistas pueden vivir en conjunto en el suelo. No se realizó ninguna determinación de razas fisiológicas de los tres hongos antagonistas empleando técnicas moleculares (CASTLE et al., 1998).

Tabla 1. Características de las cepas de los cuatro hongos antagonistas y del patógeno

\begin{tabular}{|c|c|c|c|}
\hline Código & Cepa & Procedencia & Control \\
\hline CCB-LA 101 & T. harzianum & Cañete & $\begin{array}{c}\text { Botrytis cinerea Pers.; Fr, ex Nocca \& Balb. } \\
\text { Phytophthora parasitica Dastur }\end{array}$ \\
\hline CCB-LA 102 & T. viride & Huaral & Alternaria spp. \\
\hline CCB-LA 105 & T. virens & Estados Unidos & $\begin{array}{l}\text { Rhizoctonia solani Künh } \\
\text { Crinipellis perniciosa (Stahel) Singer }\end{array}$ \\
\hline CCB-LA 114 & H. pulvinata & Ica & Dothidella ulei (P. Henn.) V. Arx \\
\hline Patógeno & C. fulvum & Ica & Patógeno \\
\hline
\end{tabular}




\section{Interacción antagonista - patógeno}

Prueba in vitro: Se evaluó la interacción de los cuatro hongos elegidos: T. harzianum, T. viride, $T$. virens y $H$. pulvinata frente al patógeno $C$. fulvum, con cinco repeticiones cada una. El método empleado para este ensayo fue el de las placas precolonizadas según Krauss et al. (1999). Se utilizaron placas de Petri de $100 \times 10 \mathrm{~mm}$ con Agar Sabouraud Dextrosa + Glucosa conteniendo a C. fulvum, de 16 días de incubación, seguidamente. Se cortaron tiras de $5 \times 25 \mathrm{~mm}$ de otras placas que contenían el crecimiento activo de los hongos antagonistas. Estas tiras fueron colocadas en forma invertida sobre un extremo de las placas que contenía al patógeno $C$. fulvum, de tal manera que entraran en contacto las hifas de las dos especies de hongos. Seguidamente, se incubaron a $24^{\circ} \mathrm{C}$ y $28^{\circ} \mathrm{C}$ con $65 \%$ de HR. Se midió el halo de crecimiento del hongo antagonista sobre el patógeno y el porcentaje de área ocupada (PAO) de la siguiente manera: cada placa precolonizada con el patógeno y su correspondiente tira de $5 \times 25 \mathrm{~mm}$ que contenía al micoparásito, fue colocada sobre una plantilla A, la cual estaba constituida por 5 columnas y 17 filas con un ancho de $5 \mathrm{~mm}$ de tal modo que se formaran celdas de $25 \mathrm{~mm}^{2}$. Esta plantilla fue colocada teniendo en cuenta que el 0 coincidiera con la tira del inóculo inicial. En la placa colonizada con la base sobre la plantilla se realizó el corte de la primera columna; seguidamente se realizaron cortes transversales con la finalidad de obtener 15 cuadrículas de $5 \times 5 \mathrm{~mm}$. Cuando se efectuó la incisión, ésta se hizo desde el margen distal del antagonista con ayuda de un bisturí estéril. Cada bloque obtenido fue transferido con ayuda de un asa bacteriológica hacia una placa vacía estéril; en la base de esta se observó una plantilla B numerada del 0 al 15 a manera de espiral. Los bloques transferidos se colocaron en orden descendente, de tal forma que esto sirvió de referencia para poder determinar el área ocupada por el antagonista. En la tapa interna de la placa, se colocó 1 disco de papel toalla estéril de 100 $\mathrm{mm}$ de diámetro, humedecido con $1 \mathrm{~mL}$ de agua destilada estéril (ADE) para brindar humedad. Posteriormente, se incubó durante 24 h, y se evaluó el desarrollo del antagonista sobre el patógeno. Las hifas del antagonista se observaron con el microscopio compuesto a un aumento total de $400 \mathrm{X}$.

Prueba in vivo: Para el experimento se utilizaron plantas de tomate de la variedad "Río Grande" de 100 días de desarrollo fenológico. Para la obtención de las plántulas de tomate, se germinaron semillas de tomate Var. Río Grande utilizando cajas de cartón de $30 \times 18 \mathrm{~cm}$; como sustrato se utilizó arena fina de río. En este sustrato se sembraron las semillas. Luego de 30 días de la germinación se transplantó a maceteros de $20 \mathrm{~cm}$ de diámetro con un sustrato mixto a base de tierra de cultivo y arena de río (1:2).

En el invernadero se colocaron seis cubos de $1 \mathrm{~m}^{3}$. Estos cubos estuvieron protegidos con una malla anti-afídica. Dentro de estos cubos se encontraron cada uno de los tratamientos. La HR fue de $75 \%$ y la temperatura fluctuó entre $28^{\circ} \mathrm{C}$ y $30^{\circ} \mathrm{C}$. La inoculación del patógeno $C$. fulvum se realizó en forma directa sobre los foliolos de la planta de tomate usando un asperjador; se utilizó una dilución de agua más Tween 80 al $0,1 \%$ que contuvieron las conidias a una concentración de $10^{5}$ esporangios $\mathrm{mL}^{-1}$. El patógeno C. fulvum no produce zoosporas. Posteriormente a la inoculación del patógeno las plantas fueron asperjadas con agua blanda, tres veces al día para favorecer la penetración del hongo patógeno. $24 \mathrm{~h}$ después de aplicar el patógeno se realizó la aplicación de los hongos antagonistas así como el tratamiento de control químico siguiendo las recomendaciones de Betalleluz (2003). Las aplicaciones de los hongos antagonistas fueron realizadas por aspersión al igual que la del patógeno. Los tratamientos para este experimento fueron seis, empleándose seis plantas por tratamiento: un químico TILT® 250 CE (Propiconazol 0,63 g IA L $^{-1}$ ), cuatro antagonistas, y un testigo (agua blanda $<180 \mathrm{mg}$ de $\mathrm{CaCO}_{3} \mathrm{~L}^{-1}$ ). La primera aplicación se realizó a las $24 \mathrm{~h}$ después de haber aplicado el patógeno. Esta aplicación se llevó a cabo de forma dirigida hacia el envés de las hojas, utilizando asperjadores de $500 \mathrm{~mL}$ de capacidad. El hongo antagonista que se encontraba en placa fue suspendido en agua, la cual contenía Tween 80 al 0,1 $\%$ para permitir encapsular a las conidias. La concentración de los hongos antagonistas para aplicar fue de $10^{8}$ esporas $\mathrm{mL}^{-1}$ siguiendo las recomendaciones de Betalleluz (2003). A cada tratamiento se le aplicaron $250 \mathrm{~mL}$ del inóculo, aproximadamente 41,6 $\mathrm{mL}$ por planta. Por ende, se emplearon 6 plantas por tratamiento. Seguidamente los tratamientos se mantuvieron en el invernadero a una temperatura promedio de 24 a $28^{\circ} \mathrm{C}$ y HR de $75 \%$.

Evaluación in vitro: Se realizaron cuatro evaluaciones que consistieron en la determinación del halo de crecimiento interdiario del antagonista sobre el patógeno. Este dato provino de la lectura de cada cuadrícula de $25 \mathrm{~mm}^{2}$, que contenía al patógeno con presencia de hifas del antagonista y la posición numérica que este ocupó en la plantilla (B). Seguidamente se multiplicó la posición numérica que ocupaba el antagonista sobre el patógeno por la distancia recorrida por éste que era de $5 \mathrm{~mm}$. Por lo tanto, al final se obtuvo el halo de crecimiento interdiario de cada antagonista, a dos temperaturas y a cuatro periodos de incubación ( 24 h a 168 h). Estos mismos datos sirvieron para determinar el porcentaje de área ocupada por el antagonista (PAO). 
Evaluación in vivo: Se realizaron cada siete días después de la inoculación del patógeno. Para la evaluación de la severidad se tomaron tres hojas del tercio medio de la planta de tomate y se estimó el nivel de daño. Se determinó la severidad de daño a nivel foliar (Tabla 2), según la escala modificada mencionada por Campbell et al. (1990). Cada tratamiento consistió en seis macetas con una planta cada una, con un total de 18 hojas por tratamiento. Con el porcentaje de área foliar dañada se procedió a estimar el área bajo la curva del progreso de la enfermedad (PE) mediante la siguiente fórmula:

$$
\begin{gathered}
n-1 \\
P E=\Sigma\left(\left[Y_{i}+Y_{i+1}\right] / 2\right)^{*}\left(t_{i+1}-t_{i}\right)
\end{gathered}
$$

donde: $\mathrm{n}=$ es el número de mediciones, $\mathrm{Y}_{\mathrm{i}}=$ el porcentaje de severidad de la enfermedad en el día de la medición $\mathrm{i}, \mathrm{t}_{\mathrm{i}}=$ el número de días después de la inoculación a la fecha de la medición i. Seguidamente se graficó el progreso de la enfermedad y se determinó el porcentaje de área reducida (Ar) usando la siguiente fórmula:

$$
\mathrm{Ar}=(\text { AAt } / \text { AAtr }) * 100
$$

Donde: AAt: área afectada del testigo.

AAtr: área afectada del tratamiento.

Tabla 2. Grados y nivel de daño causado por C. fulvum en plantas de tomate

\begin{tabular}{lc}
\hline Grado & Nivel de daño \\
\hline & $\%$ \\
0 & 0 \\
1 & 1 \\
2 & 5 \\
3 & 10 \\
4 & 25 \\
5 & 50 \\
6 & 75 \\
7 & $>75$ \\
\hline
\end{tabular}

\section{Análisis estadístico}

Pruebas in vitro: Para probar la efectividad de cada hongo antagonista se tuvo cinco repeticiones por cada tratamiento y se realizó un análisis de varianza (ANDEVA) a cada temperatura $\left(24^{\circ} \mathrm{C}\right.$ y $\left.28^{\circ} \mathrm{C}\right)$. Además, para determinar la significancia entre tratamientos se realizó la prueba de Tukey.

Pruebas en condiciones de invernadero: Se tuvo en cuenta una sola temperatura y para probar la eficiencia de cada tratamiento se tuvo 18 repeticiones, las cuales fueron sometidas a un ANDEVA, en donde los datos obtenidos se transformaron a la raíz cuadrática $(\sqrt{x}+0,5)$, para posteriormente ser sometidos a una prueba de Tukey.

\section{RESULTADOS Y DISCUSIÓN}

Se muestran los PAO de los hongos antagonistas sobre las placas precolonizadas del patógeno $C$. fulvum durante 24 a 168 h de evaluación y a una temperatura de $24{ }^{\circ} \mathrm{C}$ (Tabla 3 ). A $24{ }^{\circ} \mathrm{C}$ y a $72 \mathrm{~h}$, el PAO de T. harzianum fue estadísticamente diferente de las otras tres especies ensayadas. Así mismo se observó que a las $120 \mathrm{~h}, T$. harzianum y $T$. viride tuvieron un PAO estadísticamente igual, diferenciándose de T. virens y H. pulvinata (Tabla 3). A las 168 h se observó que T. harzianum y T. virens lograron colonizar la placa en un $100 \%$ sobre el patógeno que inicialmente fue sembrado (Tabla 3). Al realizar las medidas de crecimiento diario de los hongos antagonistas sobre el patógeno se tuvo como resultados que $T$. virens, $T$. harzianum y $T$. viride, tuvieron un promedio de crecimiento miceliar de 16 , 15,8 y $15,6 \mathrm{~mm} \mathrm{dia}^{-1}$, respectivamente, mientas el crecimiento miceliar de $H$. pulvinata fue $4,2 \mathrm{~mm} \mathrm{dia}^{-1}$.

Se observó que a una temperatura de $28{ }^{\circ} \mathrm{C}$ y a $72 \mathrm{~h}$ de evaluación, el crecimiento de $T$. harzianum y $T$. viride fue estadísticamente similar. A $120 \mathrm{~h}$, los hongos T. harzianum, T. viride y T. virens lograron ocupar el $100 \%$ del área de la placa colonizada con el patógeno inicialmente, siendo las tres especies de Trichoderma, diferentes de $H$. pulvinata (Tabla 4).

Al evaluarse la curva de crecimiento diaria de las especies ensayadas a temperatura constante de $24^{\circ} \mathrm{C}$, se obtuvo que los hongos del genero Trichoderma formaban un halo de crecimiento en forma acelerada, colonizando la placa en cinco días con 85,80 y $60 \mathrm{~mm}$ respectivamente para $T$. harzianum, $T$. viride y $T$. virens a diferencia del antagonista $H$. pulvinata y el patógeno C. fulvum, que tuvieron un desarrollo de 10,5 y $8 \mathrm{~mm}$ respectivamente Así mismo, a $28^{\circ} \mathrm{C}$ el crecimiento diario de estos hongos antagonistas se incrementó obteniéndose que en cinco días $T$. harzianum, $T$. viride y $T$. virens desarrollaron un halo miceliar de $90,88,80$ y $15 \mathrm{~mm}$ respectivamente. Resultados similares obtuvo Betalleluz (2003), lo cual corrobora que los hongos usados poseen habilidades antagónicas frente al patógeno, debido a que Trichoderma harzianum, $T$. lignorum y $T$. viride mostraron similares capacidades antagónicas (Hermosa et al., 2000; Rey et al., 2000). 
Tabla 3. Porcentaje de área ocupada (PAO) por los cuatro hongos antagonistas enfrentados al patógeno Cladosporium fulvum a $24{ }^{\circ} \mathrm{C}$

\begin{tabular}{lcccr}
\hline Hongo antagonista & $24 \mathrm{~h}$ & $72 \mathrm{~h}$ & $120 \mathrm{~h}$ & $168 \mathrm{~h}$ \\
& $\mathrm{X} \pm \mathrm{DE}$ Sig & $X \pm \mathrm{DE}$ Sig & $\mathrm{X} \pm \mathrm{DE}$ Sig & \multicolumn{1}{c}{$\mathrm{X} \pm \mathrm{DE}$ Sig } \\
\hline T. harzianum & $12,5 \pm 0 \mathrm{a}$ & $70 \pm 9,27 \mathrm{c}$ & $97,5 \pm 3,42 \mathrm{c}$ & $100 \pm 0 \mathrm{~b}$ \\
T. viride & $18,75 \pm 0 \mathrm{~b}$ & $60 \pm 3,42 \mathrm{~b}$ & $97,5 \pm 5,59 \mathrm{c}$ & $98,75 \pm 2,80 \mathrm{~b}$ \\
T. virens & $18,75 \pm 0 \mathrm{~b}$ & $55 \pm 2,80 \mathrm{~b}$ & $82,5 \pm 5,23 \mathrm{~b}$ & $100 \pm 0 \mathrm{~b}$ \\
H. pulvinata & $15 \pm 5,59 \mathrm{ab}$ & $21,25 \pm 3,42 \mathrm{a}$ & $26,25 \pm 2,80 \mathrm{a}$ & $35 \pm 5,59 \mathrm{a}$ \\
F & 6 & 76,33 & 961,905 & 534,067 \\
P & 0,006 & 0,0001 & 0,001 & 0,0001 \\
\hline
\end{tabular}

Según Tukey $(\alpha=0,05)$ letras iguales en la misma columna no difieren los porcentajes estadísticamente entre si. $\mathrm{X}=\mathrm{Promedio}$. DE $=$ Desviación estándar. $\mathrm{F}=$ Estadístico de Fisher. Sig = Significancia.

Tabla 4. Porcentaje de área ocupada (PAO) por los cuatro hongos antagonistas enfrentados al patógeno Cladosporium fulvum a $28^{\circ} \mathrm{C}$

\begin{tabular}{lcccc}
\hline $\begin{array}{l}\text { Hongo } \\
\text { antagonista }\end{array}$ & $\begin{array}{c}24 \mathrm{~h} \\
\mathrm{X} \pm \mathrm{DE} \text { Sig }\end{array}$ & $\mathrm{X} \pm$ DE Sig & $\begin{array}{c}120 \mathrm{~h} \\
\text { X DE Sig }\end{array}$ & X \pm DE Sig \\
\hline T. harzianum & $21,25 \pm 3,42 \mathrm{a}$ & $63,75 \pm 5,23 \mathrm{c}$ & $98,75 \pm 2,80 \mathrm{c}$ & $100 \pm 0 \mathrm{~b}$ \\
T. viride & $16,25 \pm 3,42 \mathrm{~b}$ & $56,25 \pm 7,65 \mathrm{bc}$ & $97,5 \pm 3,42 \mathrm{c}$ & $100 \pm 0 \mathrm{~b}$ \\
T. virens & $17,50 \pm 2,80 \mathrm{~b}$ & $52,50 \pm 3,42 \mathrm{~b}$ & $100 \pm 0 \mathrm{~b}$ & $100 \pm 0 \mathrm{~b}$ \\
H. pulvinata & $16,25 \pm 3,42 \mathrm{ab}$ & $23,75 \pm 2,80 \mathrm{a}$ & $26,25 \pm 2,80 \mathrm{a}$ & $37,5 \pm 0 \mathrm{a}$ \\
F & 2,606 & 22,647 & 5,148 & $\mathrm{NC}$ \\
$\mathrm{P}$ & 0,088 & 0,001 & 0,001 & $\mathrm{NC}$ \\
\hline
\end{tabular}

Según Tukey $(\alpha=0,05)$ letras iguales en la misma columna no difieren los porcentajes estadísticamente entre si. $X=$ Promedio. DE $=$ Desviación estándar. $\mathrm{F}=$ Estadístico de Fisher. Sig $=$ Significancia. $\mathrm{NC}=$ No calculado.

Las especies del género Trichoderma son los antagonistas más utilizados para el control de enfermedades de plantas producidas por hongos, debido a su ubicuidad, a su facilidad para ser aisladas y cultivadas, a su crecimiento rápido en gran número de sustratos y a que no atacan a plantas superiores (Grondona et al., 1997). Los mecanismos por los que las cepas del género Trichoderma desplazan al fitopatógeno son fundamentalmente de tres tipos: 1) competición directa por el espacio o por los nutrientes, 2) producción de metabolitos antibióticos, ya sea de naturaleza volátil o no volátil, y 3) parasitismo directo de determinadas especies de Trichoderma sobre el hongo fitopatógeno. Estos tres mecanismos no son excluyentes sino que actúan sinergicamente en el control de los patógenos. La importancia relativa de cada uno de ellos depende de cada pareja de antagonista-patógeno y de las condiciones ambientales (SPIEGEl y CHET, 1998; HeRmosa et al., 2000; ReY et al., 2000). Por lo tanto los resultados encontrados en la presente investigación a 24 y $28^{\circ} \mathrm{C}$ muestran que los hongos $T$. harzianum $T$. virens y $T$. viride y $H$. pulvinata ejercieron un efecto antagónico sobre el patógeno C. fulvum mediante el mecanismo de acción tipo micoparasitismo, no pudiendo observarse otro tipos de mecanismos. Betalleluz (2003) menciona que T. harzianum, T. viride, T. virens y $T$. lignorum contra $P$. infestans ejercieron un antagonismo a través de diferentes mecanismos de acción como el micoparasitismo y la antibiosis que se vieron afectados por la temperatura. Así mismo Rodríguez y ArCia (1993) mencionan que las temperaturas óptimas para el crecimiento de Trichoderma spp. se encuentra entre 25 a $30^{\circ} \mathrm{C}$. Con respecto al micoparasitismo fue el mecanismo que se pudo observar, debido que las cuatro especies ensayadas crecieron sobre el patógeno colonizándolo rápidamente y degradando sus paredes, mediante posibles actividades enzimáticas las que anteceden al micoparasitismo (REY et al., 2000; WiLLIAMs et al., 2003). Esos autores quienes mencionan que el micoparasitismo es el principal mecanismo de acción de Trichoderma, en la cual envuelve a su hospedero y secreta enzimas que degradan la pared celular, luego continua la retracción de la membrana plasmática y finalmente la desorganización del citoplasma. Las 
principales enzimas degradadoras de polímeros por parte de Trichoderma son las quitinasas, glucanasas y proteasas (REY et al., 2000). Este micoparasitismo de la pared celular se observó y corroboró al analizar las muestras en el microscopio.

Así mismo, Stefanova et al. (1999) señalan que en la actividad metabólica en el ámbito celular de las cepas de Trichoderma spp. para el control de fitopatógenos de suelo, los metabolitos producidos por los Trichoderma spp. causaron vacuolación, granulación, desintegración y lisis de la pared celular, lo cual posiblemente haya ocurrido en el ensayo de interacción entre los tres hongos del género Trichoderma y H. pulvinata contra el patógeno C. fulvum. Howell (1982) menciona que las hifas de Rhizoctonia solani Künh fueron parasitadas por $T$. virens sin mostrar evidencia de antibiosis. De la misma forma en el presente trabajo, al ser confrontadas las cuatro especies antagónicas contra el patógeno no se logró evidenciar el mecanismo de acción tipo antibiosis (CALISTRU et al., 1997). Se observó una interacción directa entre el antagonista y el patógeno siendo como consecuencia del micoparasitismo (EZZIYYANI et al., 2004).

Con respecto a la sintomatología de la enfermedad, se observó la aparición de pequeñas manchas de color amarillo sobre los haces de las hojas en el día dieciséis después de la inoculación, a una temperatura de $28 \pm 2^{\circ} \mathrm{C}$ y una HR de $75 \%$.

Los resultados de la interacción entre antagonistas y patógeno bajo condiciones de invernadero son señalados en la Tabla 5. Los resultados obtenidos entre los tratamientos son iguales estadísticamente entre la primera y quinta evaluación. Por otro lado, a los 42 días (sexta evaluación) después de la inoculación del patógeno, $T$. harzianum y el control químico fueron estadísticamente diferentes de H. pulvinata y el control. Así mismo T. harzianum, CQ, T. virens y T. viride son iguales estadísticamente. De la misma forma, HERNÁNDEZ et al. (1999), mencionan que en el control apical de la guayaba ocasionada por Dothiorella sp el uso de T. harzianum no mostró diferencias estadísticas en relación al producto químico de comparación. Otros autores han encontrado resultados que reflejan ausencia de efecto por parte de $T$. harzianum sobre la incidencia de la enfermedad pudrición carbonosa Macrophomina phaseolina (Tassi) Goid. (Cardona y Rodriguez, 2006). En contraste, EZZIYYANI et al. (2004) indicaron un alto efecto de $H$. harzianum, pues ha sido capaz de reducir en un $65 \%$ la "tristeza" causada por Phytophthora capsici en plantas de pimiento Capsicum annuum $\mathrm{L}$.
Tabla 5. Porcentaje de área foliar afectada por cladosporiosis en condiciones de invernadero

\begin{tabular}{lccccccc}
\hline \multirow{2}{*}{ Tratamiento } & Día 7 & Día 14 & Día 21 & Día 28 & Día 35 & Día 42 \\
& Eva1 & Eva 2 & Eva 3 & Eva 4 & Eva 5 & Eva 6 \\
\hline T. harzianum & 0 & 0 & 73,5 a & 74,2 a & 74,3 a & 75,2 b \\
T. viride & 0 & 0 & 75,7 a & 73,5 a & 76,7 a & 81,8 b \\
T. virens & 0 & 0 & 75,5 a & 75,7 a & 76,8 a & 79,5 b \\
H. pulvinata & 0 & 0 & 71,7 a & 73,8 a & 85,5 a & 92,0 a \\
Testigo & 0 & 0 & 74,0 a & 77,2 a & 86,0 a & 93,3 a \\
C. Químico & 0 & 0 & 73,5 a & 74,0 a & 74,2 a & 77,2 b \\
\hline
\end{tabular}

Según Tukey $(a ́=0,05)$ letras iguales en la misma columna no difieren estadísticamente.

VARGAS (1984) señala que T. harzianum en el control de Mycena citricolor fue menos eficiente en comparación con la mezcla de T. harzianum + cobox (oxicloruro de cobre), ya que se obtuvo resultados estadísticamente similares en la reducción del patógeno. Con respecto a la reducción de la enfermedad se obtuvo que T. harzianum a la sexta evaluación redujo la enfermedad en relación al testigo en un $19,35 \%$. De manera similar se observó que el tratamiento con el químico (Propiconazol) redujo la enfermedad en un $17,25 \%$, nivel promedio de control de este químico sobre $C$. fulvum. Estas diferencias aunque resulten ser mínimas numéricamente, indican que el uso de microorganismos antagónicos para el control de fitopatógenos resulta una alternativa en el control biológico de fitoenfermedades. Betalleluz (2003) señaló que $T$. harzianum no fue el hongo antagonista con mayor capacidad para reducir la enfermedad, por lo contrario el hongo que manifestó mayor capacidad de reducir la enfermedad del tizón tardío ( $P$. infestans) fue $T$. viride. Ante esto cabe señalar que Acevedo y Arcia (1988) hacen mención, con respecto a la capacidad antagonista de Trichoderma spp. que ésta dependerá de la cepa, y por ello existen aislamientos más eficientes para el control de un patógeno que para otro. Se ha evaluado el uso de hongos antagonistas en condiciones de laboratorio sobre Phytophthora infestans (Mont 1973), encontrándose que T. lignorum Persoon 1801 cubrió un $80 \%$ de la placa de precolonización en cinco días, seguido por T. harzianum con $70 \%$, T. viride con $63,3 \%$ y $T$. virens con $58,75 \%$. Además en invernadero el mejor resultado se obtuvo con $T$. viride, observándose una reducción de la enfermedad en un $62 \%$ en comparación con el químico, que redujo la enfermedad en un $88 \%$ a 10 días después de la aplicación (Betalleluz, 2003). 
Por otro lado al observar los resultados con respecto a los tratamientos $T$. viride y $T$. virens éstos muestran una reducción, de la enfermedad, respectivamente, de $12,33 \%$ y $14,79 \%$, lo cual hace suponer que los hongos antagonistas se han establecido sobre el daño ocasionado por la enfermedad. Sin embargo su agresividad en invernadero difiere de los resultados encontrados en las pruebas de laboratorio, en donde se encontró que los tres hongos del género Trichoderma tienen una acción agresiva sobre el patógeno C. fulvum llegando a crecer y colonizarlo rápidamente (Tablas 3 al 5).

H. pulvinata mostró resultados similares al testigo siendo un microorganismo antagónico deficiente para el control de la enfermedad. Esto pudiera deberse a que este antagonista no tuvo la capacidad de adaptación y crecimiento sobre el patógeno. Además se observó que el desarrollo de la enfermedad fue mayor en el testigo con 2170,23 unidades de área bajo la curva del progreso de la enfermedad (PE), seguida del tratamiento con el antagonista $H$. pulvinata, con 2119,6 unidades. Los que resultaron con menor desarrollo de la enfermedad fueron los tratamientos de T. harzianum y el control químico con 1365,43 y 1977,5 unidades, respectivamente.

Los tres hongos del género Trichoderma presentan en general un control del patógeno estadísticamente similar al producto químico propiconazole, pero diferente al del testigo (Tabla 5). Los resultados indican un nivel de control bajo como única estrategia para el control biológico e integrado de C. fulvum. Sin embargo el efecto de adelanto o atraso de la aplicación de los hongos antagonistas y la variación de la concentración inicial del inóculo $\left(10^{5}\right.$ a $10^{9}$ esporas $\left.\mathrm{mL}^{-1}\right)$ deben ser variables a ser analizadas en futuros estudios con los hongos del género Trichoderma para el control de C. fulvum.

\section{CONCLUSIONES}

1. Las temperaturas de $24^{\circ} \mathrm{C}$ y $28^{\circ} \mathrm{C}$ permitieron el crecimiento óptimo de los cuatro hongos ensayados y una buena interacción antagónica eficiente frente al patógeno Cladosporium fulvum.

2. A $24{ }^{\circ} \mathrm{C}$, Trichoderma harzianum y Trichoderma virens tuvieron un mayor porcentaje de área ocupada contra el patógeno C. fulvum.

3. A $28^{\circ} \mathrm{C}$, Trichoderma virens tuvo un mayor porcentaje de área ocupada contra el patógeno $C$. fulvum.
4. En la interacción antagonista - patógeno en condiciones in vitro se concluye que el micoparasitismo posiblemente es el principal mecanismo de acción.

5. Bajo condiciones de invernadero, el hongo antagonista más eficiente para el control de la cladosporiosis fue Trichoderma harzianum al reducir la severidad en $19,35 \%$.

\section{AGRADECIMIENTOS}

A Alfonso Lizarraga, a Yuri Calle, a Whilly Soberanis y a todos los que forman parte del equipo de trabajo del PNCB -SENASA por su apoyo desinteresado en la realización de la presente investigación.

\section{REFERENCIAS}

ACEVEDO, R.; ARCIA, A. Control biológico de Sclerotium cepivorum por Trichoderma sp In vitro. Fitopatología Venezolana, Maracay, v. 4, p.67, 1988.

APAZA, W. Principales enfermedades foliares del tomate en el Perú. Fitopatología, Lima, v.34, p.78-79, 1999.

BETALLELUZ, V. M. Control microbiológico de Phytophthora infestans en el tomate a través de hongos antagonistas (Trichoderma harzianum, T. lignorum, T. viride y Gliocladium virens) bajo condiciones controladas. Tesis (Título de Licenciado en Biología) - Universidad Nacional San Cristóbal de Huamanga, 2003.

CALISTRU, C.; MCLEAN, M.; BERJAK, P. In vitro studies on the potential for biological control of Aspergillus flavus and Fusarium moniliforme by Trichoderma species. Mycopathologia, New York, v.137, p. 115-124, 1997.

CAMPBELL, C.; MADEN, L. Introduction to plant disease epidemiology. New York: John Wiley and Sons, 1990. 532 p.

CARDONA, R.; RODRIGUEZ, H. Effects of Trichoderma harzianum fungus on the incidence of the charcoal rot disease on sesame. Revista de la Facultad de Agronomía, Maracaibo, v.23, p.44-50, 2006.

CASTLE, A.; SPERANZINI, D.; RGHEL, N.; ALM, G.; RINKER, D.; BISSETT, J. Morphological and molecular identification of Trichoderma isolates on North American mushroom farms. Applied and Environmental Microbiology, New York, v.64, p. 133-137, 1998.

DE WIT, M.J.G. P.; BRANDWAGT, B.G.; VAN DEN BURG, H.A.; CAI, X.; VAN DER HOORN, R.A.L.; DE JONG, C.F.; KLOOSTER, J. VAN' T.; DE KOCK, M.J.D.; KRUIJT, M.; LINDHOUT,W.H.; LUDERER, R.; TAKKEN, F.L.W.; WESTERINK, N.; VERVOORT, J.J.M.; JOOSTEN, M.H.A.J. The molecular basis of co-evolution between Cladosporium fulvum and tomato. Antonie Van Leeuwenhoek, New York, v. 81, p.409-412, 2002. 
ELAD, Y. Biocontrol of foliar pathogens: mechanisms and application. Communications in Agricultural and Applied Biological Sciences, Gent, v.68, p.17-24, 2003.

EZZIYYANI, M.; SÁNCHEZ, C.P.; AHMED, A.S.; REQUENA, M.E.; CANDELA, M.E. Trichoderma harzianum como biofungicida para el biocontrol de Phytophthora capsici en plantas de pimiento (Capsicum annuum L.). Anales de Biología, Murcia, v.26, p. 35-45, 2004.

GABOR, B.; WIEBE, W. Enfermedades del tomate: Guía práctica para agricultores productores y comercializadores de semilla y asesores agrícolas. Madrid: Semines Vegetable Seed, 1997. 105 p.

GIELEN, S.; AERTS, R.; SEELS, B. Biocontrol agents of Botrytis cinerea tested in climate chambers by making artificial infection on tomato leafs. Communications in Agricultural and Applied Biological Sciences, Gent, v.69 p. 631-639, 2004.

GUARRO, J.; GENÉ, J.; STCHIGEL, M. Development in fungal taxonomy. Clinical Microbiology Reviews, Washington, v.12, p. $454-500,1999$.

GRONDONA, I.; HERMOSA, R.; TEJADA, M.; GOMIS, M.D.; MATEOS, P.F.; BRIDGE, P.D.; MONTE, E.; GARCÍA-ACHA, I. Physiological and biochemical characterization of Trichoderma harzianum, a biological control agent against soilborne fungal plant pathogen. Applied and Environmental Microbiology, New York, v.63, p. 3189-3198, 1997.

HERNANDEZ, J.; ARCIA, M.; RAMIREZ, R. Comparación in vitro del control químico y biológico de Dothiorella sp., causante de la pudrición apical de la guayaba (Psidium guajava L). Revista de la Facultad de Agronomía, Maracaibo, v. 16, p.S49-S55, 1999.

HONÉE, G.; VAN DEN ACKERVEKEN, G. F. J. M. ; VAN DEN BROEK, H. W. J.; COZIJNSEN, T.J.; JOOSTEN, M.H. A. J. KOOMAN-GERSMANN, M.; VERVOORT, J.; GELSANG, R.; VOSSEN, P.; WUBBEN, J.P.; DE WIT, P.J.G.M. Molecular characterization of the interaction between the fungal pathogen Cladosporium fulvum and tomato. Euphytica, Washington, v.79, p.219-225, 1994. (Historical Archive)

HERMOSA, M.R.; GRONDONA, I.; ITURRIAGA, E.A.; J.M. DIAZ-MINGUEZ; CASTRO, C; MONTES, E.; GARCÍA-ACHA. Molecular characterization and identification of biocontrol isolates of Trichoderma spp. Applied and Environmental Microbiology, Washington, v.66, p. 1890-1898, 2000.

HOWELL, C. Effect of Gliocladium virens on Pythium ultimun, Rhizoctonia solani and Damping - Off of cotton seedings. Phytophatology, St. Paul, v.72, p.496-498, 1982.

IANNACONE, J.; MURRUGARRA, Y. Efecto del nim y rotenona en las poblaciones de Tuta absoluta (Meyrick) (Lepidoptera: Gelechiidae) en dos especies de áfidos (Homoptera: Aphididae) en el cultivo de Tomate en Ica, Perú. Folia Entomológica Mexicana, Capital Federal, v. 41, p. 119-128, 2002.

IANNACONE, J.; REYES, M.U. Efecto en las poblaciones de Bemisia tabaci (Homoptera: Aleyrodidae) y Liriomyza huidobrensis (Diptera: Agromyzidae) por los insecticidas botánicos neem y rotenona en el cultivo de tomate en el Perú. Revista Colombiana de Entomología, Bogotá, v. 27, 147-152, 2001.
JOOSTEN, M.; DE WIT, P. The tomato-Cladosporium fulvum interaction: A versatile experimental system to study plantpathogen interactions. Annual Review of Phytopathology, California, v.37, p.335-367, 1999.

KLEIN, D.; EVELEIGH, E. Ecology of Trichoderma. In: KUBICEK, C.P., HARMAN G. E. (Ed.). Trichoderma and Gliocladium: Basic biology, taxonomy and genetics. London: Taylor y Francis, 1998. vol.1., p. 53-74.

KRAUSS, U.;SOBERANIS, W.; MATTEWS, P. The use of antagonistic mixtures in biocontrol. In: U. KRAUSS AND H. PRAKASH (Ed.). Research methodology in Biocontrol of plant diseases: Workshop Manual. Turrialba, Costa Rica: Catie, 1999. p. 112-121.

LIECKFELDT, E.; SAMUELS, G.J.; NIRENBERG, H.I.; PETRINI, O. A morphological and molecular perspective of Trichoderma viride: is it one or two species. Applied and Environmental Microbiology, Washington, v.65, p. 2418-2428, 1999.

LO, C.; NELSON, E.; HARMAN, G. E. Improved biocontrol efficacy of Trichoderma harzianum 1295-22 for foliar phases of turf diseases by use of spray application. Plant Disease, St. Paul, v.81, p.1132-1138, 1997.

MARTÍNEZ, B.; FERNÁNDEZ, L.; SOLANO, T. Antagonismo de cepas de Trichoderma frente a hongos fitopatógenos de la caña de azúcar, tomate y tabaco. Cultivos Tropicales, La Habana, v.15, p. 5, 1994.

MERCK. Microbiology Manual. Merck Eurolab: Ed. Frankfurter, 2000. p. 407.

PAPAVIZAS, C. Trichoderma and Gliocladium: biology, ecology, and potential for biocontrol. Annual Review of Phytopathology, Palo Alto, v. 23, p. 23-54, 1985.

PERESSE, M.; LE PICARD, D. Hansfordia pulvinata, mycoparasite destructeur du Cladosporium fulvum. Mycopathologia, New York, v. 71, p. 23-30, 1980. (Historical Archive)

REY, M.; DELGADO-JARANA, J.; RINCÓN, A. M.; LIMÓN, M.A..; BENÍTEZ T. Mejora de cepas de Trichoderma para su empleo como funguicidas. Revista Iberoamericana de Micología, Bilbao, v.17, p.S31-S36, 2000.

RODRÍGUEZ, V. Efecto antagónico y biocontrolador de algunos microorganismos saprófitos contra Rhizoctonia solani un fitopatógeno causante del (Damping off) en plantas de tomate. 2002, 105 p. Tesis para optar el grado de Magíster en Microbiología - Universidad Nacional Mayor de San Marcos, Lima, 2002.

RODRÍGUEZ, I.; ARCIA, A. Caracterización fisiológica (temperatura, $\mathrm{pH}$ y luz) de 12 aislamientos de Trichoderma spp., in vitro. Fitopatología Venezolana, Maracay, v. 6, p. 53, 1993.

SAMUELS, G. Trichoderma a guide to identification and biology. Beltsville, Maryland: Agricultural States Department of Agriculture. USA, 2004. 40p.

SANTAMARINA, M.P.; ROSELLO, J.; LLACER, R.; SANCHIS, V. Antagonistic activity of Penicillium oxalicum Corrie and Thom, Penicillium decumbens Thom and Trichoderma harzianum Rifai isolates against fungi, bacteria and insects in vitro. Revista Iberoamericana de Micología, Bilbao, v.19, p.99, 2002. 
SARIAH, M.; CHOO, C.W.; ZAKARIA, H.; NORIHAN, M.S. Quantification and characterisation of Trichoderma spp. from different ecosystems. Mycopathologia, New York, v.159, p. 113-117, 2005.

SELITRENNIKOFF, C.P. Antifungal proteins. Applied and environmental Microbiology, Washington, v.67, p. 28832894,2001

SPIEGEL, Y.; CHET, I. Evaluation of Trichoderma spp. as a Biocontrol Agent Against Soilborne Fungi and Plant-parasitic Nematodes in Israel. Integrated Pest Management Reviews, New York, v.3, p. 169-175, 1998.

STEFANOVA, M.; LEIVA, A.; LARRINAGA, L.; CORONADO, M.F. Metabolic activity of Trichoderma spp. isolates for a control of soilborne phytopathogenic fungi. Revista de la Facultad de Agronomía, Maracaibo, v.16, p. 509-516, 1999.
THOMMA, B. P.H.J.; PETER VAN ESSE, H.; CROUS, P.W.; DE WIT, P.J.G.M. Cladosporium fulvum (syn. Passalora fulva), a highly specialized plant pathogen as a model for functional studies on plant pathogenic Mycosphaerellaceae. Molecular Plant Pathology, Bristol, v. 6, p. 379-393, 2005.

VARGAS, E. Interacción de tratamiento biológico y químico en el combate del ojo de gallo (Mycena citricolor) en el cafeto. Agronomía Costarricense, San José, v.8, p. 91-97, 1984.

WILLIAMS, J.; CLARKSON, J.M.; MILLS, P.R.; COOPER, R.M. A selective médium for quantitative reisolation of Trichoderma harzianum from Agaricus bisporus compost. Applied and Environmental Microbiology, Washington, v.69, p. 4190-4191, 2003. 